Pedro Guevara, Cultura ciudadana y TICs

\title{
INCORPORACIÓN DE TIC A LA EDUCACIÓN CIUDADANA COMO ESTRATEGIA PARA PROMOVER LA CULTURA DE LA SEGURIDAD VIAL Y PREVENCIÓN DE ACCIDENTES EN AMÉRICA LATINA
}

\author{
Tic Incorporation To Citizen Education As A Strategy To Promote Road Safety Culture \\ And Accident Prevention In Latin-American
}

Pedro Guevara S. ${ }^{1}$, Eduardo Carrillo $Z^{2}$.

Recibido para publicación: 20 de octubre de 2012 - Aceptado para publicación: 11 de diciembre de 2012

\section{RESUMEN}

Actualmente la utilización de Tecnologías de la Información y la Comunicación (TIC) proporciona un espacio virtual para apoyar la interacción entre personas que quieran aprender en Internet sobre cualquier actividad o temática de interés específico. El diseño de una plataforma educativa Web 2.0 brinda los elementos tecnológicos necesarios para que conductores, peatones, motociclistas y autoridades de tránsito en general, tengan un ambiente donde puedan comunicarse, intercambiar experiencias, consultar dudas, aprender sobre seguridad vial preventiva, señales y normas de tránsito. Esto sirve a la ciudadanía como un espacio pedagógico que aporte conocimientos en la formación de una cultura vial para mejorar el comportamiento en las vías y evitar accidentes de tránsito.

\section{Palabras claves}

Seguridad vial, señales de tránsito, normas de tránsito, TIC, accidentes de tránsito.

\section{ABSTRACT}

Currently the uses of TIC (Information Technology and Communication) provide a virtual space to support the interaction between people. People interested in learning online about any activity or themes of specific interest. The design of an educational platform Web 2.0 provides all the technological elements so all drivers, pedestrians, and motor vehicles authorities can have a space. Trough this space they will have the opportunity to communicate, exchange experiences, and solve

${ }^{1}$ Especialista en Informática y Telemática, Magíster en Software Libre. Miembro Grupo de Investigación SOCRATES Universidad de Córdoba. Correo electrónico: pedrogsguevara@hotmail.com

2 doctorado Tecnología de La Inf Computac y Las Comunicac Universitat De Valencia, docente universidad autónoma de Bucaramanga, ecarrill [at] unab.edu.co. 
Pedro Guevara, Cultura ciudadana y TICs

questions. Also, they can learn about road safety, traffic signs, and traffic laws. It helps the community to create pedagogic spaces to provide knowledge about road culture. In addition, it will help to improve the behavior on the roads and eventually avoid traffic accidents.

Keywords: Road safety, traffic signs, traffic law, TIC and traffic accidents

\section{INTRODUCCIÓN}

Los accidentes de tránsito diariamente causan la muerte a miles de personas en todo el mundo, muertes que se pueden evitar realizando acciones para mejorar la seguridad vial[7]. Se requiere promover entre los conductores de cualquier tipo de vehículo, un cambio de conducta y una cultura preventiva, a través de espacios donde se publiquen temas relacionados con la prevención de riesgos en las carreteras. La accidentalidad en las vías se ha convertido en un problema de salud pública para la sociedad actual, según la Organización Mundial de la Salud (OMS) se tiene previsto que para el año 2030 los accidentes de tránsito sean la quinta causa de muerte de las personas a nivel mundial (Cámara de Comercio de Bogotá, n.d).

Desde el punto de vista de la psicología de la seguridad, se indica que para la prevención de accidentes de tránsito se debe promover entre los conductores, un cambio de conducta y cultura preventiva [1] En Colombia las principales causas de accidentes de tránsito en el año 2010 fueron: el exceso de velocidad, la impudencia de los peatones y la conducta irresponsable de conducir bajo los efectos del alcohol. Estas causas se asocian a fallas humanas, por esta razón es importante, además de los controles de las autoridades, hacer énfasis en el cambio de actitud y responsabilidad de las personas a través de la educación vial para prevenir los accidentes.

Existen en el ámbito internacional y nacional entidades que contribuyen con el mejoramiento de la seguridad vial mediante la realización de campañas preventivas. Estas instituciones cuentan con portales Web que buscan concientizar a las personas sobre la responsabilidad al conducir y promueven la cultura vial preventiva para evitar accidentes de tránsito. Entre las principales instituciones se encuentran: La Dirección de Tránsito de
España (DGT), la Fundación RACC, distraction.gov, el Fondo de Prevención Vial, el Ministerio de Transporte (Colombia), Comisión Nacional de Seguridad de Tránsito de Chile, Dirección Nacional de Vialidad Argentina, Comisión de Tránsito del Ecuador, entre otros. Estas instituciones en sus sitios Web promueven la seguridad vial y el respeto por las normas de tránsito, pero no permiten la participación activa de los usuarios para que puedan agregar contenidos, compartir experiencias, recursos o comentarios. Estos sitios solamente se limitan a publicar información relacionada con las diferentes campañas viales que realizan para educar a las personas; la interacción entre las instituciones y los usuarios es mínima. En este sentido las personas que acceden a estos sitios solamente consultan información, se informan. Las instituciones promueven principalmente sus servicios, pero no centran la atención en temas relevantes de seguridad vial, normas de tránsito y prevención de accidentes.

Las TIC'S se constituyen en herramientas tecnológicas, disponibles para facilitar la comunicación de las personas en los diferentes campos de la actividad humana como: la educación, comercio, industria, medicina, cultura, etc. Actualmente el Estado colombiano promueve el acceso y uso de TIC, por medio de la ley 1341 del 30 de julio de 2009 [14]

Sin duda alguna las TIC, a través del uso de Internet proporcionan los recursos para desarrollar procesos de aprendizaje. Entre los elementos tecnológicos disponibles para facilitar la comunicación de las personas, se encuentran las aplicaciones Web 2.0, que ofrecen como principal ventaja la colaboración entre los usuarios; en un espacio donde pueden aprender, generar conocimientos y compartirlos [2]. 
Por esta razón se hace necesario utilizar herramientas Web 2.0 para crear una plataforma educativa, que apoyada en el uso de TIC fomente la cultura vial preventiva y agrupe en una comunidad a conductores, peatones, motociclistas y autoridades de tránsito en general, para que compartan experiencias, recursos y participen en temas de discusión relacionados con la prevención de accidentes, normas y señales de tránsito.

\section{ESTADO DEL ARTE}

\subsection{Seguridad vial y prevención de accidentes de tránsito}

Las personas a cualquier edad pueden tener el riesgo de sufrir accidentes de tránsito, esto implica inculcarles desde niños la formación vial y los hábitos de auto protección, para que sean conscientes de utilizar los sistemas de seguridad que traen los vehículos.

Los jóvenes también se ven involucrados en accidentes de tránsito por la poca experiencia en el ejercicio de conducir y al consumo de alcohol, a éstos se suman los peatones que son los actores menos tenidos en cuenta al momento de realizar las campañas preventivas que promueven las instituciones encargadas de la seguridad vial[9], es indispensable incluir la educación vial en los colegios y escuelas de conducción para promover, desde temprana edad, en las personas el respeto por las normas de tránsito y la cultura vial responsable.

Muchos de los accidentes se producen por fallas humanas, la distracción de conductores y peatones es una situación que hace parte de esta problemática. Entre las principales causas de distracción en los conductores se encuentran: Realizar actividades diferentes a las de conducir; la somnolencia producto del cansancio; perder por segundos la atención de la vía; escuchar la radio, hablar por teléfono celular; conversar con otro pasajero; pensar en otras actividades y separar las manos del volante para sostener objetos
El consumo de alcohol también es un factor de riesgos a tener en cuenta, este es un determinante de en gran parte de los accidentes de tránsito en el mundo [11]. De igual manera la fatiga, porque los conductores pierden la capacidad de reacción; pueden pestañear 0 dormirse y esto genera una situación de riesgo, es una de las formas de perder la atención en la vía [10].

\subsection{Accidentalidad en Colombia y Latinoamérica}

Al mirar las cifras de los últimos años en Colombia, se puede evidenciar el problema de la seguridad en sus carreteras y vías. El Ministerio de Transporte en el año 2008 reportó que se presentaron 185.733 accidentes de tránsito en carreteras, de los cuales 5.670 personas resultaron muertas[8]. De igual forma, el Fondo de Prevención Vial reportó que en el año 2009 ocurrieron 175.872 accidentes de tránsito en las carreteras colombianas, de los cuales 39.138 personas sufrieron lesiones graves y 5.635 resultaron muertas. Además, son la primera causa de muerte en niños entre 5 y 14 años, también son la segunda causa de fallecimiento en personas entre 15 y 45 [15] La Policía Nacional de Tránsito y Transporte reportó para el año 2010 un total de 6.275 accidentes, la mayoría ocurridos en las horas de la noche, dejando un saldo trágico de 1.086 muertos. Las principales causas de estos accidentes de tránsito fueron: Exceso de velocidad, conducir en estado de embriaguez, cruzar la calle sin observar y no respetar las señales de tránsito (RCN La Radio, 2010). Estas cifras demuestran que Colombia presenta uno de los más altos índices de accidentalidad de América Latina.

El Estado colombiano para el año 2019 busca fomentar, a través de la cultura ciudadana, estrategias que impulsen el cambio cultural de las personas. Una de las metas que plantea cumplir el Gobierno está relacionada con la seguridad vial, promoviendo el cambio de comportamiento en las vías y el respeto por las normas y señales de tránsito [4]

En América Latina el panorama no es el más alentador, desde hace algunos años se ha 
Pedro Guevara, Cultura ciudadana y TICs

considerado los accidentes de tránsito como un problema de salud pública que genera costos sociales y económicos [17].

En América Latina y el Caribe, las cifras son alarmantes, el número de víctimas mortales por accidentes de tránsito asciende a 17 muertes por cada 100.000; los accidentes también son la principal causa de muerte en las personas que tienen entre 15 y 29 años y la segunda causa entre niños y jóvenes menores de 14 años. Para el año 2020 las cifras podrían aumentar de 17 a 31 muertes por cada 100.000 [12]

Buscando mejorar el problema de la accidentalidad, instituciones como el Banco Interamericano de Desarrollo y la Organización Mundial de la Salud entre sus planes de acción incluyen proyectos, campañas y estrategias para mejorar la seguridad vial en la región de América Latina y el Caribe durante los próximos años. Teniendo en cuenta que son pocos los países que han logrado reducir las muertes por accidentes de tránsito en los últimos 10 años, la Organización Panamericana de la Salud recomendó a los gobiernos de América Latina recopilar mayor información de los siniestros, con el propósito de generar mejores políticas públicas que contribuyan a reducir la mortalidad por esta causa (Voz de América, 2010).

\subsection{Instituciones que promueven la seguridad y educación vial}

Existen en Latinoamérica instituciones gubernamentales y no gubernamentales, algunas sin ánimo de lucro, encargadas de promover la seguridad vial en cada uno de sus países. A continuación se describen las entidades relacionadas con la seguridad y educación vial para prevenir accidentes de tránsito:

En Colombia, se encuentra el Fondo de Prevención Vial, institución no gubernamental sin ánimo de lucro, que tiene como objetivo educar a las personas a través de campañas sobre seguridad vial y respeto de las normas de tránsito. Trabaja con entidades estatales en la realización de campañas para capacitar a conductores sobre normas tránsito y seguridad en las vías, generando en la población una cultura que permita reducir los accidentes de tránsito.

La Dirección Nacional de Vialidad Argentina se encarga principalmente de la gestión vial, pero presenta en su portal Web enlaces sobre educación vial en donde se explica el uso de las señales de tránsito, mediante animaciones.

El Instituto de Seguridad y Educación Vial de Argentina (ISEV), es una institución privada dedicada a la investigación en materia de tránsito, educación y seguridad vial para brindar asistencia técnica al sector público y privado. CONASET es la Comisión Nacional de Seguridad de Tránsito de Chile, entidad encargada del control carretero y la seguridad de tránsito.

El Instituto de Tránsito Brasil (ITB), es una organización sin ánimo de lucro que busca promover la ética ciudadana para ayudar a construir con la responsabilidad de todas las personas un tránsito seguro. Hace énfasis en el respeto de las normas de tránsito y la educación para sensibilizar a la gente en seguridad vial.

En Costa Rica el Ministerio de Obras Públicas y Transporte, ofrece el Consejo de Seguridad Vial (COSEVI), organización encargada de promover en la sociedad una cultura de seguridad vial, buscando mejorar calidad de vida de los habitantes.

Se puede resaltar también a nivel mundial el esfuerzo que realizan entidades sin ánimo de lucro como la Fundación RACC, encargada de fomentar la cultura automovilística entre los conductores. Realiza estudios y campañas de formación sobre seguridad vial preventiva y movilidad, contribuyendo a la reducción de los accidentes en las carreteras de España; además, busca proteger el medio ambiente difundiendo buenas prácticas de movilidad referente a este tema.

Carreteras Seguras es una Campaña mundial de seguridad vial para prevenir accidentes de 
tránsito en las carreteras del mundo. También distraction.gov es un portal Web oficial del Departamento de Transportes de los Estados Unidos para concientizar a las personas del gran problema de conducir distraídos.

Se pueden destacar algunos problemas comunes en la mayoría de los portales Web de las instituciones descritas anteriormente. Los usuarios casi siempre consultan información, pero no agregan contenidos, además faltan canales de comunicación y herramientas para expresarse y opinar. A lo anterior se suma que varias de estas instituciones ofrecen otros servicios adicionales, que desvían la atención en los temas relevantes de seguridad vial, normas de tránsito y la prevención de accidentes.

\subsection{Plataforma Web 2.0 como contribución a la prevención de accidentes de tránsito}

El término Web 2.0 hace referencia a un nuevo enfoque que utiliza Internet como plataforma de trabajo, bajo un esquema que se basa en la participación de los usuarios. Existen plataformas de comunicación que integran diferentes tecnologías Web 2.0, facilitando la edición, publicación e intercambio de información, permitiendo que las personas creen sus propios contenidos, interactúen socialmente y compartan recursos; este fenómeno es conocido con el nombre de "Social Media" (Wikipedia, n.d). Estos medios de comunicación social generalmente se encuentran disponibles sin ningún costo y son fáciles de usar.

Los conceptos que enriquecen este fenómeno son: la generación de contenidos por parte del usuario, sindicación de los contenidos, redes sociales, entre otros [3]. Proporciona soporte a la sociedad de la información y el conocimiento, ofreciendo un nuevo modelo que funciona alrededor de Internet. Cuenta con una comunidad de usuarios que está creciendo, por la incorporación de personas que han comenzado a utilizar Internet debido a las jornadas de alfabetización digital, realizada por administraciones públicas [5].

Después de investigar sobre los conceptos relacionados con la seguridad vial, conducción preventiva, señales y normas de tránsito; además de estudiar las características de los portales Web de instituciones que promueven la educación vial en Latinoamérica y otras partes del mundo, se crea una plataforma educativa Web 2.0 como estrategia para fomentar la cultura vial y prevenir accidentes de tránsito (Ver Figura 1).

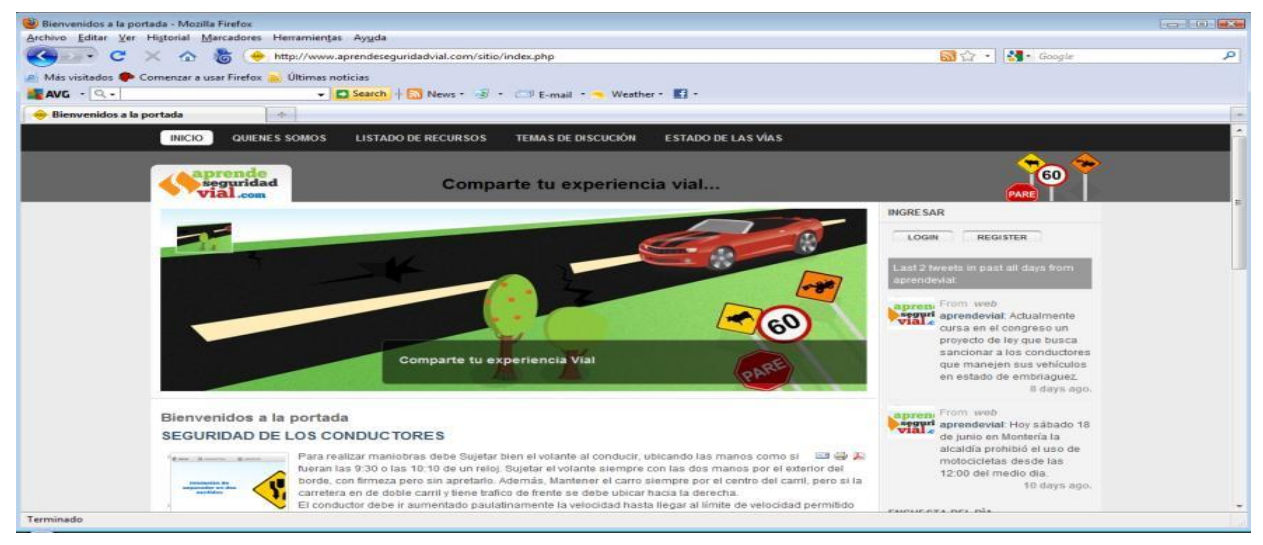

Figura 1. Página principal

El nombre asignado al portal es principales funcionalidades del sitio se
aprendeseguridadvial.com. Entre las encuentran: Un banco de recursos de


aprendizaje con guías informativas; foro de discusión para tratar temáticas referentes a normas de tránsito y prevención vial; consultas del estado de las vías; enlace a las redes sociales; compartir recursos y cualquier tipo de experiencia vial.

Los visitantes pueden encontrar guías de aprendizaje informativas, con diseños intuitivos que facilitan entender los conceptos referentes a los temas de prevención vial, normas y señales de tránsito (Ver figura 2). Igualmente se cuenta con un banco de recursos de aprendizaje, estos recursos se organizan por categorías asociadas a las temáticas tratadas y pueden ser descargados por cualquier persona.

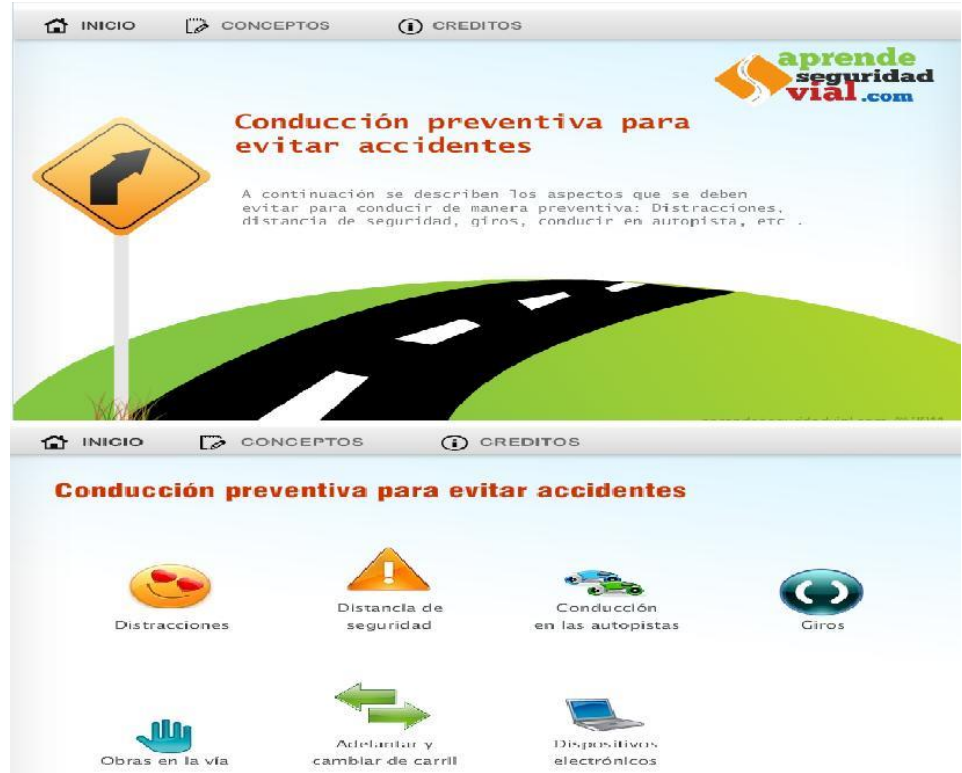

Figura 2. Portada de una guía de aprendizaje y Opciones guía de aprendizaje

Los usuarios tienen tres foros temáticos en donde pueden interactuar: un foro sobre normas de tránsito, otro relacionado con seguridad y prevención vial. Y un tercer foro para compartir preguntas. Los usuarios registrados podrán acceder y participar en los foros de discusión o realizar sus preguntas.

Además, cuentan con un espacio para compartir experiencias y recursos de aprendizaje, así como poder comentar sobre las experiencias viales y recursos que publiquen otros usuarios. Existe un módulo de gestión del estado de las vías, de utilidad para los viajeros, donde pueden consultar sobre el estado de la infraestructura vial, informándose si una vía está cerrada, con derrumbes o se están realizando obras.

Los visitantes pueden seguir los últimos tweets publicados en la cuenta oficial de Twitter del sitio, buscar los artículos publicados y votar la encuesta del día. Las funcionalidades descritas facilitan la interacción y participación activa de los usuarios para que puedan agregar contenidos, recursos, compartir experiencias o comentarios.

\section{CONCLUSIÓNES}

Está claro que los accidentes de tránsito son un problema mundial del que no escapan los 
países de América Latina y el Caribe. Por esta razón instituciones reconocidas a nivel mundial como: La Organización Mundial de Salud, el Banco Interamericano de Desarrollo, Naciones Unidas y otras instituciones comprometidas con la prevención vial, promueven entre las administraciones públicas y la sociedad, planes de acción con campañas preventivas para reducir las muertes por accidentes de tránsito en los próximos 10 años.

A través de la aprobación del nuevo Código de Tránsito, con la ley 1383 de 2010 que reforma algunos artículos del antiguo Código Nacional de Transporte (ley 769 de 2002), el Congreso de la República de Colombia quiere inculcar un cambio de cultura en los conductores, pasajeros y peatones para que el tránsito vehicular en las vías del país sea seguro.

Es importante resaltar que en los países de la región las autoridades de tránsito realizan controles y campañas preventivas para evitar accidentes, pero son los ciudadanos quienes tienen la responsabilidad de fomentar una cultura vial preventiva. En este sentido todos debemos conocer y respetar las normas de tránsito, y promover la seguridad vial en las carreteras o vías del país.

Muchas veces los accidentes de tránsito se producen por fallas en la infraestructura física de las vías, en este sentido la falta de planeación en algunas ciudades de Colombia ha generado un desarrollo desordenado, ejemplo de esto son las mejoras logísticas realizadas a los sistemas de transporte masivo, sin antes haber realizado las adecuaciones requeridas en las vías.

El uso de las nuevas Tecnologías de la Información y la Comunicación proporcionan un espacio virtual para que las personas compartan y aprendan en Internet sobre temas que fomenten la cultura vial responsable, en este sentido resulta interesante la utilización de una plataforma Web 2.0 como estrategia educativa que ayude en la prevención de accidentes de tránsito. $\mathrm{La}$ herramienta aprendeseguridadvial.com es un aporte tecnológico para a la sociedad, en donde las personas pondrán aprender sobre seguridad vial, señales y normas de tránsito, con el propósito de generar una cultura vial entre los conductores, motociclistas, peatones y autoridades encargadas del tránsito.

Puede ser utilizada en campañas de prevención vial por entidades gubernamentales, colegios, empresas de transporte, escuelas de conducción y entidades comprometidas con el tema de la accidentalidad. Es importante crear conciencia ciudadana para que las personas tengan en cuenta los peligros que existen cuando se viaja en carretera y se tomen las medidas preventivas necesarias para evitar accidentes de tránsito.

\section{REFERENCIAS}

[1]. Asunción Valverde, Luis S. (2005). Psicología de la seguridad y prevención de riesgos de accidentes en carretera. Universidad Nacional Federico Villarreal, facultad de psicología Lima - Perú.

[2]. Barkley, Elizabeth; Cross, K. Patricia y Howell Major, Claire. (2007). Técnicas de aprendizaje colaborativo. Madrid: Ministerio de Educación y Ciencia/Morata.

[3]. Cobo Romaní, Cristóbal y Pardo Kuklinski, Hugo. (2007). PLANETA WEB 2.0. Inteligencia colectiva $O$ medios fast food. Barcelona, España / México. Página 15.

[4]. Departamento Nacional de Planeación, (2006). Fomentar la cultura ciudadana Visión Colombia II Centenario. Imprenta Nacional de Colombia. Pagina 45-48.

[5]. Fumero, Antonio; Roca, Genís y Sáez Vacas, Fernando. (2007). Web 2.0. Madrid, España: Fundación Orange - Omán Impresores.

[6]. Gras Pérez, María E.; Planes Pedra, Montserrat y Font-Mayolas, Silvia. (2008). La distracción de los conductores: un riesgo no percibido. Fundación RACC, Barcelona, España: Digital Screen, S.L, Fundación RACC. 
[7]. McMahon, Kate y Ward, David. (2007). Make Roads Safe. Commission for Global Road Safety y FIA Foundation.

[8]. Ministerio de Transporte. (2009). Oficina Asesora de Planeación - Anuario estadístico del trasporte en Colombia 2009.

[9]. Muñoz Medina, Miguel M; Cogollos Paja, María y Zaragoza Ramírez, Aniceto. (2006). Libro Verde de la Seguridad Vial. Madrid, España: Asociación Española de la Carretera.

[10]. Rey de Castro Mújica, Jorge. (2003). Accidentes de tránsito en carreteras e hipersomnia durante la conducción. ¿Es frecuente en nuestro medio? la evidencia periodística. Rev Med Hered, vol.14, no.2, p.69-73.

[11]. Vázquez Pedrouzo, Rodolfo A. (2004). Causas de los accidentes de tránsito desde una visión de la medicina social. El binomio alcohol-tránsito. Revista Médica del Uruguay.

[12]. Banco Interamericano de Desarrollo. Plan de Acción para mejorar seguridad vial en América Latina y el Caribe. Consultada el 20 de julio de 2011.

http://www.iadb.org/es/noticias/comunicadosde-prensa/2010-03-19/seguridad-vial-deamerica-latina-banco-interamericano-dedesarrollo-reunion-anual-2010,6707.html

[13]. Cámara de Comercio de Bogotá Universidad de los Andes (n.d), Boletín de accidentalidad vial No. 2. Consultada el 16 de julio de 2011. http://camara.ccb.org.co/documentos/4419_b oletin_de_accidentalidad.pdf

[14]. Edutecno.org (2009), Ley de TIC. Consultada el 17 de julio de 2011. http://edutecno.org/2009/08/colombia-ley-detic-2009/

[15]. Fondo de Prevención Vial (2010), Campaña inteligencia vial. Consultada el 18 de junio de
2010.

http://www.epidemiadeexcusas.com/home.ht $\mathrm{ml}$

[16]. Fundación RACC (2007), cada 3 minutos muere un niño en las carreteras del mundo. Consultada el 16 de julio de 2011. http://w3.racc.es/index.php?mod=fundacion\& $\mathrm{mem}=$ EPDetalle\&relmenu=31\&id=2208

[17]. Organización Panamericana de la Salud (2004), Informe mundial sobre prevención de los traumatismos causados por el tránsito. Consultada el 20 de julio de 2011. http://www.paho.org/spanish/dd/pub/resumen _informe_mundial_traumatismos.pdf 